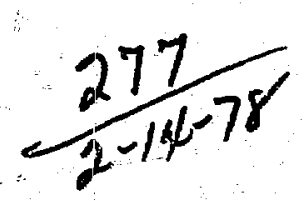

Lh. 1833

UCRL-52373

\title{
FIELD-REVERSAL EXPERIMENTS IN THE MIRROR FUSION TEST FACILITY (MFTF)
}

MHSTER

J. W. Shearer

W. C. Condit

December 7, 1977

Prepared for U.S. Energy Research \& Development Administration under contract No. W-7405-Eng-48

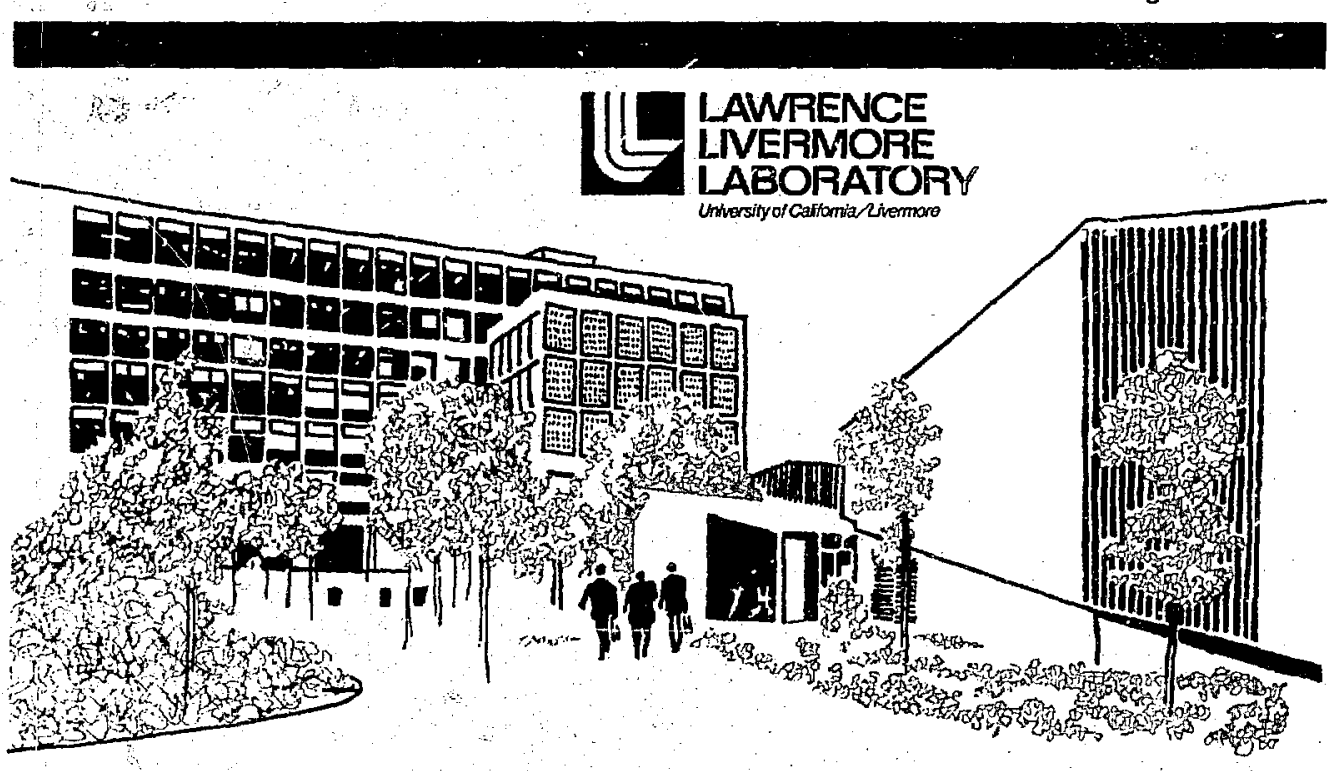




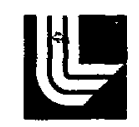

\title{
LAWRENCE LVERMORE LABORATORY
}

Universityol Catomia/Livermore,Calitomia/94550

\section{UCRL-52373 \\ FIELD-REVERSAL EXPERIMENTS IN THE MIRROR FUSION TEST FACILITY (MFTF)}

\author{
J. W. Shearer \\ W. C. Condit
}

MS. date: December 7, 1977

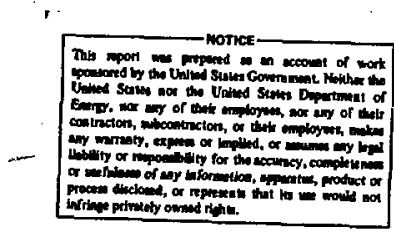




\section{FIELD-REVERSAL EXPERIMENTS IN THE MIRROR FUSION TEST FACILITY (MFTF)}

\section{ABSTRACT}

We have begun detailed consideration of several aspects of a field-reversal experiment in the Mirror Fusion Test Facility (MFTF): Model calculations have provided some plausible parameters for a field-reversed deuterium plasma in the MFTF, and a buildup calculation indicates that the MFTF neutral-beam system is marginally sufficient to achieve field reversal by neuiral injection alone. However, the many uncertainties indicate the need for further research and development on alternate buildup methods. A discussion of experimental objectives is presented and important diagnostics are listed. The range of parameter space accessible with the MFTF magnet design is explored, and we find that with proper aiming of the neutral beams, meaningful experiments can be performed to advance towards these objectives. Finally, we point out that if we achieve enhanced $n \tau$ confinement by means of field reversal, then quasi-steady-state operation of MFTF is conceivable.

\section{INTRODUCTION}

In this report, we describe the preliminary formulation of plans for experiments on field reversal in the Mirror Fusion Teat Facility (MFTF). This large facility is scheduled to be brought on-line in $1981,1,2$ and conventional mirror experiments are planned for the initial operations. ${ }^{3}$ Early on, however, perthaps as coon as 1982, field-reverued mirror experiments are deairable because of the attractive possibilities offered by field-reversed mirror (FRM) reactors, 4,5

Our first step in this report is a survey of possible field-reverced configurations in the prevent MFTF design. For this purpose, we use the same calculational model that was developed in the reactor studies 4,6 and find some useful possibilities. Secondly, we examine the neutral-beam capability. for buildup of such configurations and find that it is only marginally sufficient. Further knowledge, both theoretical and experimental, is needed to clarify this buildup problem.

For both steady-state and buildup conditions, we list a number of physics questions that must be ad- dressed by experiment. One of the most important of these, the stability question, depends on the shape of the magnetic field. We compare the magnetic field of the present MFTF magnet design with the 2XIIB field and with the fields in recent experiments to determine the stability of relativistic electron rings "; we find roughly the same range of quadrupole field strengths in the three cases. 2XIIB experiments in progress are thus directly relevant to similar MFTF experiments and may provide the data needed to evaluate the effect of the MFTF quadrupole field component on MFTF fieldreversal experiments.

This report is obviously only a "fint cut," since both relevant theory and experiments are in preliminary stages of development. The final section describes some implications for MFTF operations, if a successful field-reversed plasma with high plasma containment $\mathrm{n}$ r can be achieved. We find that quasj-steady-state operation might be possible and that it could provide information which could be extrapolated to designs for FRM reactors. 
It should be immediately pointed out that the beam current required for this model is much lower than that needed for a conventional mirror plasma. The reason is that the field-reversed model has a longer particle escape time. (If it didn't, there would be no reason to reverse the field.) The implications of this difference will be discussed later. Other consequences of the longer particle escape time are that the ratio of ion energy to neutral-beam energy $\mathrm{E}_{\mathrm{i}} / \mathrm{E}_{\mathrm{B}}$ is lower than for a conventional mirror plasma, and the electron energy $\mathrm{E}_{\mathrm{v}}$ is higher.

The specified MFTF beam-extraction potential is $80 \mathrm{kV}$. For the field-reversed case, the computed overall plasma dimensions using $80 \mathrm{kV}$ beams are a little smaller than the conventional mirror plasma defined for the first MFTF experiment. In particular, the $23.6-\mathrm{cm}$ plasma radius and the $10.7-\mathrm{kG}$ vacuum field are buth clearly compatible with the MFTF design parameters. ${ }^{1-3}$

In the second set of computer runs, we ask what the field-reversed plasma conditions will be using the 80-keV beam energy and a fixed geometry corresponding to a fixed neutral-beam alignment. We were guided by scaling laws ${ }^{8}$ derived from the computer model equations ${ }^{6}$ under the assumption that $T_{i} \propto E_{B}$ and $T_{e} \propto E_{B}$ :

$$
\begin{aligned}
& S \equiv \frac{a}{\rho_{1}} \propto \frac{a B_{0}}{E_{B}^{1 / 2}}, \\
& n_{0} \propto \frac{\beta B_{0}^{2}}{E_{B}}, \\
& \tau \propto a^{2} B_{0}^{2} E_{B}^{1 / 2}, \\
& M \propto \beta B_{0}^{2} \frac{(\sigma)}{E_{B}}, \text { and } \\
& \left(1-c^{-2 M}\right) I_{B} \propto\left(\frac{R}{a}\right)\left(\frac{L}{a}\right) \beta^{2} \frac{B_{0}^{2}}{E_{B}^{5 / 2}},
\end{aligned}
$$

where $S=a / \rho_{i}, \quad(\sigma)$ is the mean ionization cross soction of the beam, and the cther quantities are as defined above. In the first computer calculations (Fis. 1), $\beta, S$, and $M$ are held fixed and the other parameter are allowed to vary as a function of $E_{B}$. In the ecoond set of runs, $E_{B}$ is fixed and $\beta, S$, and $M$ are the independent variables. Each combination of $\beta, S$, and $M$ is chosen to as to hold the geometry constant (minor radius a is held constant; and the shape is fixed as before). The two sets of computer runs overlap at the values $E_{B}=80 \mathrm{kV}, \mathrm{B}=10.7 \mathrm{kG}$.
Figure 2 shows the results of the second set of computer runs; they agree well with the scaling laws [Eqs. (9) through (13)]. We find that the higher field capability of the MFTF allows one to explore the possibilities of field-reversed plasmas that are many Larmor radii in diameter (see the $R_{p} / \rho_{j}$ vs B curve). The scaling law predicts an $\mathrm{n} r$ exceeding $5 \times 10^{13}$ near $B=20 \mathrm{kG}$. However, the plasma absorption efficiency $\eta$ is so high in this case that shielding of the inner regions by the outer plasma may prevent the plasma doughnut from reaching the "fat" shape that was assumed.

Table 1 is a summary of the plasma parameters calculated by this model for three cases.* The parameter $I_{B} \tau_{p}$ is of interest to the buildup calculations; it represents the total charge injection required to suddenly produce this equilibrium state. The values for $n \tau_{p}$ are admittedly optimistic; they are based on the assumption that there is no additional plasma on the open field lines and on the diffusion parameter assumptions of Eq. (8). The Larmor radius $\rho_{\mathrm{j}}$ of a deuterium ion is given in the code calculations by

$$
\rho_{\mathrm{i}}=0.2 \mathrm{~T}_{\mathrm{i}}^{\mathrm{t} / 2} / \mathrm{B}
$$

\begin{tabular}{|c|c|c|c|}
\hline Purneter & $\mathbf{A}$ & $\mathbf{B}$ & C \\
\hline$\beta$ & 15 & $\mathbf{I S}$ & 2.0 \\
\hline $\mathbf{s}=\mathbf{a} \boldsymbol{\rho}_{\mathbf{i}}$ & 3. & 4.24 & 4.24 \\
\hline $\mathbf{M}$ & 1.0 & 2.0 & $\mathbf{2 . 6 7}$ \\
\hline $\mathbf{B}(\mathbf{k G})$ & 10.7 & 15.7 & 15.7 \\
\hline $\mathbf{I}_{\mathbf{B}}(\mathbf{A})$ & $22.0^{n}$ & $30.3^{\mathbf{*}}$ & $48.8^{\circ}$ \\
\hline $\mathbf{P}_{\mathbf{B}}(\mathrm{MW})$ & $1.76^{a}$ & $2.42^{*}$ & $3.90^{\circ}$ \\
\hline$P_{\text {In }}(\mathbf{M W})$ & 1.00 & 1.89 & 3.55 \\
\hline $\mathbf{E}_{\mathbf{i}}(\mathbf{k e V})$ & 28.7 & 27.3 & 27.3 \\
\hline $\mathrm{E}_{\mathrm{e}}(\mathrm{ceV})$ & 9.7 & 11.6 & 11.5 \\
\hline$n\left(10^{14} \mathrm{~cm}^{-3}\right)$ & 1.68 & 3.53 & 4.72 \\
\hline$T_{p}(\theta)$ & 0.103 & 0.092 & 0.069 \\
\hline$a(\mathrm{~m})$ & 7.86 & 7.44 & 7.42 \\
\hline $\mathbf{R}_{p}=\mathbf{R}+\mathbf{a}=3 \mathbf{2}(\mathrm{cm})$ & 23.6 & 22.3 & 22.3 \\
\hline$L=3 R=6 a(\mathrm{~cm})$ & 47.2 & 44.7 & 44.7 \\
\hline$I_{B} T_{p}(C)$ & $2.3^{*}$ & $2.8^{2}$ & $3.4^{\mathrm{a}}$ \\
\hline $\mathrm{n}_{\mathrm{p}}(1013, \mathrm{~cm}-3)$ & 1.7 & 3.2 & 3.2 \\
\hline
\end{tabular}

Table 1. Three computed steady-tate models for $E_{B}=80 \mathrm{kV},(R / a)=2,(L / R)=3$, and tengentiv injection into $D_{2}$ plame.

values have been corrected by the absorption approximation deacribed in the Appendix to this roport.

This same equilibrium calculation can also be compared with results based on the 2 XIIB geometry, where the ratio $\mathrm{K}_{\mathrm{p}} / p_{\mathrm{i}}$ is much amaller than we have anumed here. This work is in protreas and will not be diecused here. 

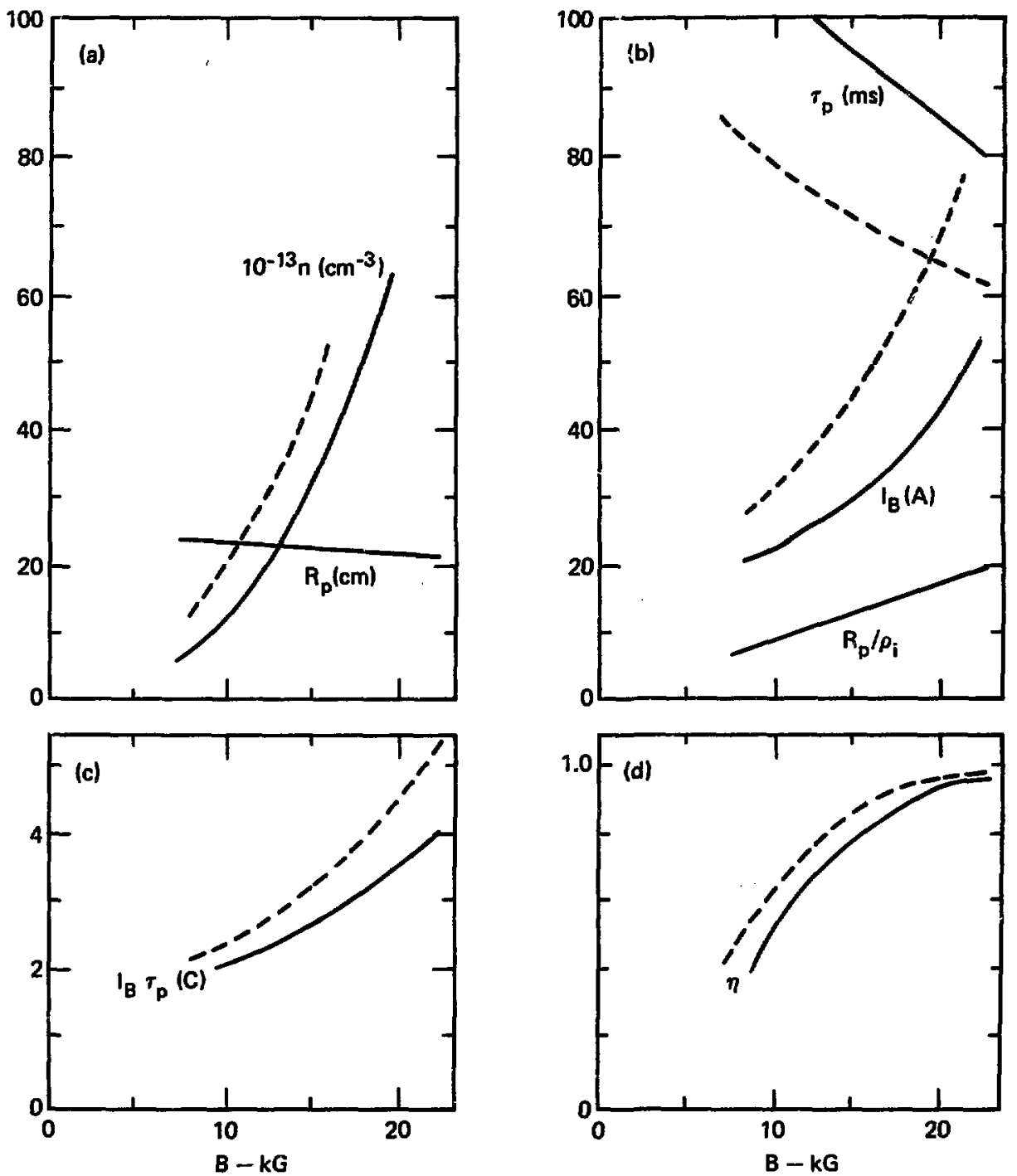

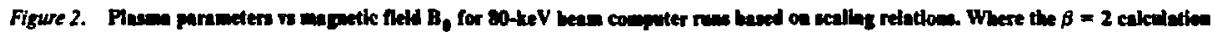
differs from the $\beta=15$ case, it is thown by a dotted line.

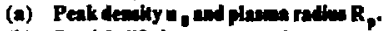

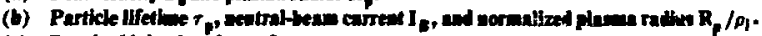

(c) Requred injection chare I $T_{\text {. }}$.

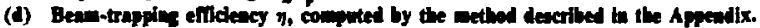


where $T_{i}$ is the ion temperature in $\mathrm{eV}$, and $B$ is the magnetic field in $\mathrm{kG}$. In these runs, we find that $T_{i}$ $\approx \mathrm{E}_{\mathrm{B}} / 4$; thus, a freshly injected $80-\mathrm{kV}$ ion will have a Larmor radius $\rho_{B}=2 \rho_{i}=5.28 \mathrm{~cm}$ in the $10.7 \cdot \mathrm{kG}$ vacuum field. Of course, in the actual field-reversed plasma, the orbits are more complicated and span even more space. 9,10

Our computer calculations have thus established that the MFTF can support interesting, field- reversed plasmas for the study of stability, lifetime, and similar questions. By varying the magnetic field and the beam focusing, one can span a range of scaled plasma size $R / \rho_{j}$ that runs from 2 XIIB $(\sim 3)$ to the proposed reactor designs (10 to ?0). The required sustaining current is much lower than is actually available-the buildup requirement, however, is larger and will be described in the next section.

\section{BUILDUP CALCULATIONS}

We are attempting to build up a field-reversed mirror plasma on the 2XIIB experiment; if successful, we can extrapolate the result to the MFTF geometry.

We begin by outlining (in Fig. 3) the four stages of buildup by means of neutral beams. Stage (a) is the injection of an initial target plasma, shown in the figure as plasma from streaming-plasma guns. Stage (b) is the buildup of a "conventional" $\beta \approx 1$ mirror plasma by intense neutral-beam bombardment of the target plasma; that plasma is stabilized by additional plasma frum streaming-plasma guns. Both stages (a) and (b) have been successfully achieved in the 2XIIB experiment.

In Fig. 3, stage (c) is the transition stage in which sufficient net positive-ion current is produced to reverse the center field and produce closed field lines. The inner plasma, on the closed field lines, should have a larger containment parameter $n \tau$ and a higher electron temperature $T_{E}$. The outer plasma, on the open field lines, would have the conventional mirror containment $n r$. Stage (d) is the final desired state, which is obtained by lowering the incident flux of the reatral beams and of the stabilizing plasma as far as possible.

Buildup in the MFTF device along open field lines is described by Stallard and Rensink ${ }^{11}$; we apply their method to the plasmas of Table 1 . To do this, we first correct the total absorption parameter M [Eq. (7)] as follows:

$$
\frac{M\left(1, E_{B}\right)}{M(\beta, 80)}=\frac{1}{\beta} \frac{\sigma_{T}}{\sigma_{80}}
$$

This correction is necessary because the open field line buildup calculation is only valid for $\beta<I$ and because the cross-section correction is necessary for the calculation of the effects of the $20-\mathrm{keV}$ buildup beams.
The beam-trapping efficiency $\eta$ is then estimated from the equation derived in the Appendix [Eq. (A12)]:

$$
\eta=\frac{\sigma_{i}\left(1-e^{-2 M}\right)}{\sigma_{i}+\sigma_{x} e^{-M}} .
$$

The condition that there be sufficient total current to achieve buildup is then

$\eta_{20} I_{20}+\eta_{80} I_{80}>\frac{\text { en }}{\tau_{p}} V_{p}=\frac{e n^{2} V_{p}}{n \tau_{p}}$,

where the left-hand side is the sum of the trapped currents, and the right-hand side is the hot-ion loss current, $\tau_{p}$ is the particle-loss time, and the plasma volume $V_{p}$ is estimated from Table 1 :

$$
V_{p}=\pi R_{p}^{2} L_{p}
$$

As in the previous calculations, ${ }^{11} \mathrm{n} \tau_{\mathrm{p}}$ is scaled from $2 X$ IIIB results:

$$
\frac{\mathrm{n} \tau}{(\mathrm{n} \tau)_{0}}=\left(\frac{\mathrm{B}}{\mathrm{B}_{0}}\right)\left(\frac{L_{\mathrm{p}}}{L_{0}} \frac{\beta}{\beta_{0}} \frac{\Delta_{0}}{\Delta}\right)^{1 / 2}
$$

where $(n \tau)_{0}=7 \times 10^{10}, B_{0}=6.7 \mathrm{kG}, L_{0}=40 \mathrm{~cm}$, $\beta_{0}=0.5$, and $\Delta_{0}=0.02$. The parameter $\Delta$ is the ratio of the density of the warm, stabilizing plasma to that of the hot plasma. Also, this scaling uses the fact that electron drag is the predominant mechanism for particle loss. For the MFTF plasma, we obtain the-magnetic field $B$ and the plasma length $L_{p}$ from Table $l, \beta=1$, and we estimate $\Delta$ from Fig. B-6 of Ref. 3. In our case, $R_{p} / \rho_{j}=9$ to 13; thus, $\Delta \approx 0.005$.

In Table 2, we apply Eqs. (15) through (19) to the cases of Table 1. When we use the specified total 


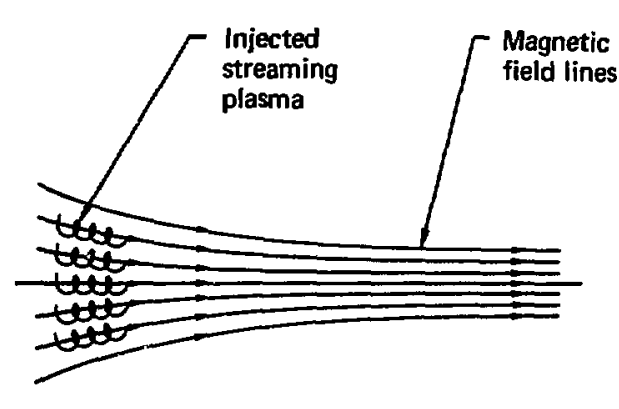

(a)

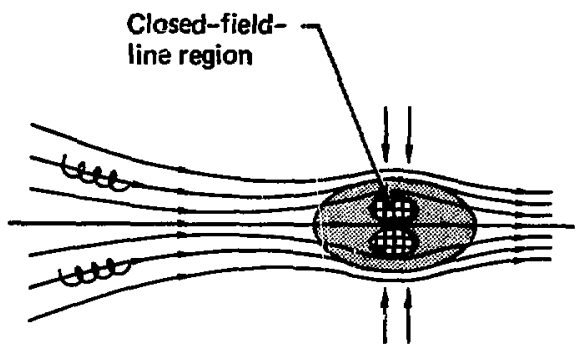

(c)
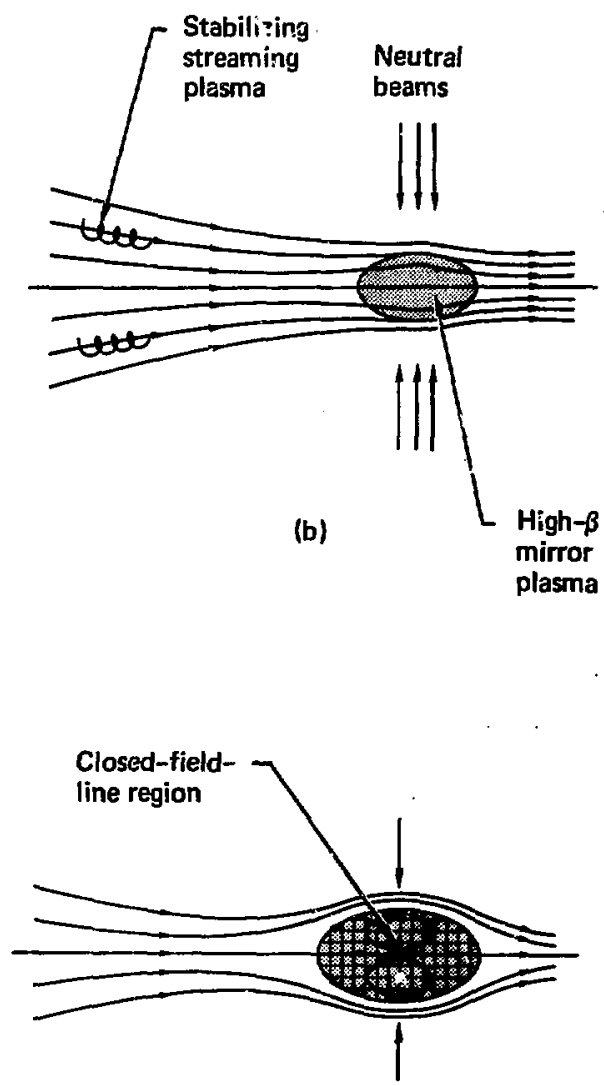

(d)

Figure 3. Four stages in the milldup of field-reversed nirror clasa:

(a) Strean Ijection.

(b) Convertional eisror plasma.

(c) Tranition atege.

(d) Fiek-reversed pluma.

MFTF beam current capability of $1000 \mathrm{~A}$ at $20 \mathrm{kV}$ plus $750 \mathrm{~A}$ at $80 \mathrm{ky}$, the buildup capability and the total current requirement are almost equal, within estimated uncertainties.

A somewhat smaller $\mathcal{E}=1$ plasma (radius $\mathrm{R}_{\mathrm{p}}=$ 15 , for example) would have required less buildup current and would be a more conservative choice. Such a calculation was done earlier, ${ }^{11}$ and it did demonstrate a greater buildup current surplus. The smaller plasma, of course, requires a tighter focus.
More detailed studies of this part of the buildup problem are needed. The details of plasma geometry, secondary neutrals, and the fractional mix of $20-\mathrm{kV}$ and $80-\mathrm{kV}$ beams should be studied.

Stages (c) and (d) of the neutral-beam buildup scenario are under active study, both experimentally and theoretically, and it is not yet possible to present scaling relations in this $\triangle B / B$ regime. There is considerable theoretical concern about the possibility that large electron currents in low-field 
Table 2. Buildup calculations to wehieve $\mathrm{g}=1$ plams for the reometries of Table 1 .

\begin{tabular}{|c|c|c|}
\hline Parneter & A & $B$ and $C$ \\
\hline B (kG) & 10.7 & 15.7 \\
\hline$V_{p}$ (itres) (Eq. 18) & 81.7 & 69.8 \\
\hline n $\left(1014 \operatorname{con}^{-3}\right)(a t \beta=1)$ & 1.12 & 2.35 \\
\hline $\operatorname{mr}\left(1011 \mathrm{~cm}^{-3.1-1)}\left(\mathrm{E}_{q} \cdot 19\right)\right.$ & 3 & $\mathbf{s}$ \\
\hline \multicolumn{3}{|l|}{ 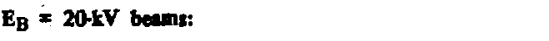 } \\
\hline$M(E q .15)$ & 1.61 & 3.22 \\
\hline$\eta$ (Eq. 16) & $\mathbf{0 . 3 3}$ & 0.64 \\
\hline$T_{20}(A)$ & 1,000 & 1,000 \\
\hline \multicolumn{3}{|l|}{$E_{B}=80-5 V$ beans: } \\
\hline $\mathbf{M}(\mathbf{E q}, 15)$ & 0.67 & 1.33 \\
\hline$\eta(\mathrm{Eq}, 16)$ & $\mathbf{0 . 4 3}$ & 0.68 \\
\hline$I_{80}(A)$ & 750 & 750 \\
\hline $\begin{array}{l}\eta_{20} I_{20}+\eta_{80} I_{80} \\
\text { (lefthand dide of Eq. } 17\end{array}$ & 652 & 1,150 \\
\hline $\begin{array}{l}\operatorname{en}^{2} V_{p} / n_{p} \\
\text { (righthand ide of Eq. 17) }\end{array}$ & 547 & 1,233 \\
\hline Current maryin (A) & +105 & -83 \\
\hline
\end{tabular}

regions would inhibit the transition to field reversal. A small component of high-Z ions (e.g., helium) would produce a so-called Ohkawa current that may solve the problem. A theoretical analysis of this matter is in progress. ${ }^{12}$

An alternate injection method, use of the Intense Pulsed Ion-Neutral Source (IPINS), was recently proposed and is being investigated at Lawrence Livermore Laboratory under the aegis of the Applied Plasma Physics group of the Department of Energy's Division of Magnetic Fusion Energy. The objective of this program is, briefly, to produce neutral-beam currents of the order of $\mathrm{kA} / \mathrm{cm}^{2}$ for a time of the order of $1 \mu \mathrm{s}$. The system uses a reflex triode ion source to provide the high intensities. ${ }^{13}$ The total charge required is estimated from the steady-state code calculations by multiplying the sustaining current $I_{B}$ by the loss time $\tau_{p} ;$ it is plotted in Figs. 1 and 2 as the injection charge $I_{B^{\tau}}$. For the nominal Table 1 examples, one finds $\hat{i}_{B} \tau_{p}=2$ to $4 \mathrm{C}$, if we assume that the IPINS with an average energy equal to the average ion energy ( $30 \mathrm{kV}$ ) is required to do the whole job of injection. Higherenergy ions would lower the required charge.

Overall, it appears that the neutral-beam capability being planned for the MFTF is just sufficient to produce field-reversed plasmas. The many uncertainties in our present theoretical knowledge of field reversal provide a stimulus to further research and development on alternate buildup methods of which the IPINS is one example.

\section{EXPERIMENTAL OBJECTIVES}

In the following discussion, we assume that the MFTF is an operating facility which has been previously "broken in" with conventional mirror experiments. 2,11 Then, for field-reversal experiments, the experimental plans can be naturally divided into three broad classes: startup and scaling, steady-state and plasma conditions, and reactorrelated experiments.

Startup and scaling experiments proposed here are modeled on the 2XIIB field-reversal experiments. A suggested sequence of experiments is as follows:

- Try startup with narrowly focused, $20-\mathrm{keV}$ beams alone. The buildup calculations suggest that field reversal is problematical.

- Try again with both $20-\mathrm{keV}$ and $80-\mathrm{keV}$ beams. Here the buildup calculations indicate that field reversal may be attained.

- Add the pulsed IPINS option to expand the buildup capability.
Each of these options would be carried out over a range of magnetic field values and with various focusing options in order to sample a variety of values of the parameters $S=a / \rho_{i}$ and the aspect ratio $R / a$. Methods for detecting field reversal are described later, in the diagnostics section.

The second class of experiments concerns the behavior of the plasma in a field-reversed steady state. These experiments must address the following questions:

- Is the field-reversed plasma stable, or does it break up again soon after buildup? Are electron current effects observed, or is plasma behavior described by an ions-only model (i.e., the SUPERLAYER code)?

- What is the range of parameters for which stability is observed? Here we mean especially the size and shape of the plasma $\left(R_{p} / \rho_{\mathrm{i}}, R / a, L / a\right)$ and also the shape of the magnet field. 
- What is $\mathrm{n} r$ and what are the plasma loss rates? Ways to study this include turning off the beams in order to observe the decay of the plasma parameters and observing the minimum beam current required to maintain the steady state.

- Are there gross changes in behavior at various characteristic times after buildup? Such times include $r_{\mathrm{ie}}$ (electron drag), $\tau_{\mathrm{ii}}$ (ion-ion equilibration), and $\tau_{n}$ (resistive diffusion).

- What are the effects of impurities? Are there pumping problems and similar cold-boundary effects?

From the experimental evaluation of such questions, one could draw conclusions concerning the relevant plasma instabilities and the proper transport matrices to use for energy and particle losses. Thus, the assumptions of the FRM reactor calculations $^{5}$ can be tested, and modified if necessary.

The third class of experiments would be more closely related to reactors; an example would be cold-gas refueling tests. These wivuld be later work, and their planning depends on the results of the experiments in the first two classes.

This concludes our evaluation of the experimental objectives. We emphasize that we anticipate a considerable evolution and development of ideas and concepts before actual experimentation can begin.

\section{IMPORTANT DIAGNOSTICS}

Next, we briefly discuss the diagnostics techniques that should be the most useful for fieldreversal experiments in MFTF.

Ideally; a direct measurement of magnetic field B in the center of the plasma is the most straightforward diagnostic. In a proposed method for doing this at one instant of time, the Zeeman effect in the optical spectrum of a beam of helium ions is used. The magnetic field is deduced from optical measurements involving two polarized laser beams. This method will be tested soon on 2XIIB; if successful, it can be used for the MFTF experiment. A small helium neutral-beam source, two dye laser beams, and an optical fluorescence-detection channel will be required.

Faraday rotation of a polarized light beam is another promising diagnostic. This technique measures $\int B$ nd $\ell$ along the optical path. Careful choice of optical path, combined with simultaneous interferometric measurements of $\int$ nd $\ell$, should permit the unfolding of information concerning the magnetic field $B$. This method will also be tested on 2XIIB. For MFTF, we will re-evaluate whether to use the HCN laser at $\lambda=337 \mu \mathrm{m}$ (as on 2XIIB) or to choose another laser such as $\mathrm{CO}_{2}$ at $\lambda=10.6 \mu \mathrm{m}$.

Diagnostic loop measurements outside the plasma are simple and will undoubtedly be done; however, the effect of field reversal at the center of the plasma is obscured by other effects, such as the details of the shape of the distribution of the plasma currents.

A different diagnostics approach is the indirect one-to look for changes in the plasma inat can be attributed to the effects of field reversa'.. A good example of this is variation in the clectron temperature $T_{e} ; T_{e}$ should increase within the closed field lines, and its variation can be measured at a few selected times and places by Thomson scattering of ruby laser light (as is now done on 2Xl1B). Continuous monitoring of $T_{c}$ is conceivable with $x$ ray detectors of bremsstrahlung radiation; this should be simpler on MFTF than on 2XIIB because of expected higher values of $T_{E}$.

Another indirect method is to measure the confinement parameter $\mathrm{nr}$, as described in the discussion on experimental objectives. As shown by the steady-state calculations earlier in this report, a dramatically lower steady-state neutral-beam current is all that may be required to maintain a field-reversed plasma.

A final indirect approach relies on a characteristic of the spatial distribution of a field-reversed plasma: it should have a midplane density peak that is well off-axis. This might be detected by unfolding measurements of $\int \mathrm{nd} \ell$ (interferometric or beam absorption techniques). It might also be seen by the proposed neutron pinhole camera diagnostics. 14 


\section{MAGNETIC FIELD SHAPE}

A preliminary assessment suggests that the MFTF coil design allows us to explore a significant range of parameters for field-reversed plasmas that are built up and sustained by neutral beams.

We compare the MFTF coil magnetic field with the 2XIIB magnetic field as it is used in current experiments. The total magnetic field along the axis $B(0, z)$ and along the midplane axes $B(R, 0)$ is normalized to the center field $\mathrm{B}_{00}$. The exial and radial dimensions are normalized to the Larmor radius $\rho_{\text {: }}$ of the average energy ions ( $27 \mathrm{kV}$ for MFTF and $9 \mathrm{kV}$ for $2 \mathrm{XIIB})$. The change in magnetic field $\Delta \mathrm{B} / \mathrm{B}_{00}$ is plotted in Figs. 4 and $S$ vs $z / \rho_{\mathrm{i}}$ and $R / \rho_{\mathrm{i}}$, respectively. The specific cases chosen for comparison are the $\beta=1.5, \mathrm{~B}=15.7-\mathrm{kG}$ calculation (Tables 1 and 2) for MFTF, and the 2XIIB "gas box" experiment, which has the highest beta yet reported. 15

Figure 4 shows the fractional change in total magnetic field us axial distance for the two cases. One finds that the MFTF field is flatter than the 2XIIB field, which is reasonable because it was deliberately designed for experiments with largersized plasmas. The highest value of the ordinate of Fig. 4 is unity, corresponding to a mirror ratio of $\mathbf{R}_{\mathrm{m}}=2$; this is much higher than the lower limit of the vacuum mirror ratio that was used in the initial FRM reactor studies ${ }^{5}\left(R_{m}>1.0001\right.$ there). However, for a better comparison with these studies, one should pick a point on the axis of 2XIlB or MFTF which is closer to the plasma. But

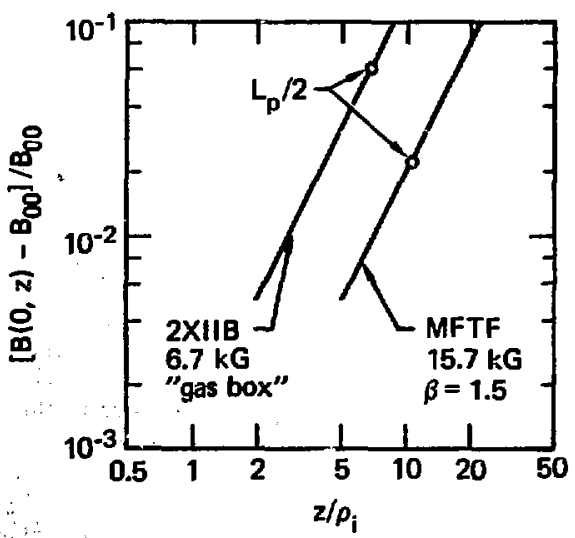

Floure 4. Conparioca betwee the 2XIIB and MFTF ve sum

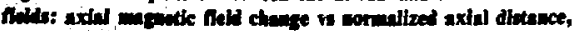

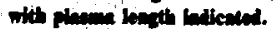

even in that case, choosing $1<Z / \rho_{\mathrm{i}} \leqslant 10$ leads to an axial magnetic field change that is 10 to 100 times greater than the lower limit of the FRM reactor study. No upper limit is given to this parameter in that study; the authors imply that it is limited by economic rather than physical considerations. In any case, the MFTF design is slightly closer to the FRM reactor design than is the 2XIIB experiment.

Figure 5 shows the radial variation of the fractional chringe in total magnetic field, corresponding to the quadrupole component of the coils. In addition, a-comparison is made with the parameter range of the Cornell electron-ring quadrupole experiments. ' As in Fig. 4, the MFTF field is flatter than the 2XIIB case. Although the normalized radii are different, the quadrupole strengths of both MFTF and 2XIIB are within the range studied with the electron rings.

The quadrupole field is necessary for minimum-B stabilization of magnetohydrodynamic (MHD) modes in the open-field-line mirror plasmas, but its role in the field-reverser case is more ambiguous. Recent results from the electron-ring quadrupole experiment at Cornell ${ }^{7}$ are interesting, even though there are obvious differences between an electron ring decaying in a partially-ionized plasma and a plasma that is fully ionized, has a multi-Larmor radius, and is sustained by ne:ttral beams. The electron experiments ${ }^{7}$ show that sufficient quadrupole field stabilizes very weak, non-field-:aversed electron rings $(\triangle B / B<0.1)$ against the precessional

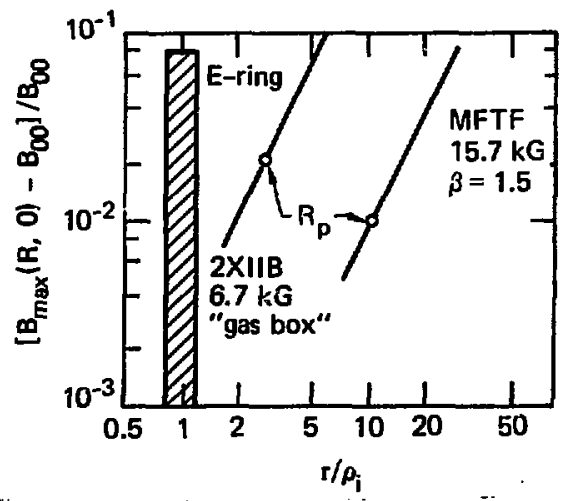

Figure 5. Comparion between the 2 XIiB an MFTF vacuma fieth: raltal magaetic lield change (neialy the quadrapole conponext) vs connaliaed nodial distunce, with plasmi radiss indicated. The hulched har sows the parneter naye of the Cornell electronriag (E-ring) quadropole experiments (aee text). 
mode. On the other hand, slightly stronger rings $(\Delta \mathrm{B} / \mathrm{B}>0.25)$ decay more rapidly with than without quadrupole fields-the decay rate being proportional to the strength of the quadrupole field component. The hypothesis of orbit 11 resonance ef- fects has been advanced to explain the enhanced decay of the axis-encircling electrons in the Cornell experiment. ${ }^{7}$ This hypothesis is being investigated by computer calcu! -tions at LLL. 12

\section{SOME CONSEQUENCES OF A SUCCESSFUL FIELD-REVERSAL EXPERIMENT ON MFTF OPERATING PROCEDURES}

Clearly, a favorable evolution of theoretical and experimental understanding is needed to achieve a field-reversed plasma in any facility. For example, Post's orbit theory ${ }^{13}$ (admittedly only twodimensional) predicts stat field reversal can occur only when the plasma is large $\left(R / \rho_{i}>3\right)$, which favors MFTF over 2 XIIB. If surface effects (charge exchange or radial heat loss) are limiting, MFTF will have a significant advantage over 2XIIB because the larger MFTF plasma has a smaller surface-to-volume ratio.

Should field reversal be achieved under the conditions given by the sustaining equilibrium calculation described above, this would have important inplications for the programming of the $80-\mathrm{keV}$ neutral be ans. During buildup, all 20 to 24 beams are on; after field reversal is achieved, only 20 to 60 A may be required, and that can be supplied by just two ..ndules.

If all the modules are left on, the plasma would continue to try to grow, as shown by SUPERLAYER ${ }^{9}$ calculations, unless or until it is disrupted by an instability. It may be necessary to throttle back the beams before this happens. This throttling could be programmed in ahead of time; however, it may be more desirable to use a feedback control based on some field-reversal diagnostic such as a diamagnetic loop or a Faraday rotation measurement.

Consider again the hoped-for steady-state, fieldreversed plasma. The $n \tau$ is $10^{13}$ instead of $10^{12}$, the loss-power fluxes are lower than in the standard MFTF design by a factor of 10 . Thus, it is conceivable that the field-reversed plasma might be sustained for a long time. As each pair of $80-\mathrm{keV}$ modules reaches its design limit of $30 \mathrm{~s}$, another pair is programmed in to sustain the field-reversed plasma for another $30 \mathrm{~s}$. Assuming that there are 22 operating modules (11 pairs), one would cycle through all the $80-\mathrm{kV}$ neutral beams in 5-1/2 min. Because the power supply design calls for a 5 -min repetition puriod, in principle one can start the cycle all over again. Thus one can envision the possibility of long time operation of a field-reversed mirror experiment in MFTF.

However, the present 30 -s water-ccoled beamdump design is awkwardly matched 12 this operational possibility. The water is stored in tanks, and can only be cycled once through the beam dur ps; for this throttled-back operation, water would be available only for about $30 \times 11$ s (5$1 / 2 \mathrm{~min}$ ). It would be better to reinvestigate the possibility of heat exchangers or direct conversion for this application.

There are indeed many more immediate milestones to be attained before detailed consideration of such a sustained experiment is needed. It is important, however, to keep this optimistic possibility in mind. The plasma size and the magnetic field are only factors of 2 to 4 less than the corresponding parameters for the prototype optimum FRM reactor, ${ }^{5}$ and they are factors of 2 to 4 greater than the present 2 XIIB experiment. If it becomes possible to run the MFTF in this longterm, sustained, field-reversed mode, then such experiments would be an important intermediate step towards a prototype FRM rec.ucor. 


\section{SUMMARY}

This preliminary survey of a possible fieldreversed mirror plasma experiment in the MFTF is based on the assumption that neutral beams are capable of both building up and sustaining such a plasma. We find that the MFTF design is suitable for such field-reversal experiments, with the possible exception of a marginal nestral-beam buildup capability. Successful production of a wellcontained $\left(\mathrm{n} r \approx 10^{13}\right.$ ) field-reversed plasma would open up the possibility of long-term, steady-state operation of the MFTF.

\section{Acknowledgments}

The authors have benefited from discussions with T. K. Fowler, F. H. Coensgen, C. C. Damm, R. S. Devoto, and D. S. Prono.

\section{REFERENCES}

1. "A Mirror Fusion Test Facility," in Energy and Technology Review, H. P. Shay, Scientific Editor, Lawrence Livermore Laboratory, Rept. UCRL-52000-77-10 (1977)

2. Mirror Experimental Facility Project Management Plan, Lawrence Livermore Laboratory, Rept. UCRL52213 (1977).

3. MX Major Project Proposal, F. H. Coensgen, Editor, Lawrence Livermore Laboratory, Rept. LLLProp-142 (1976).

4. T. K. Fowler and R. F. Post, The U.S. Mirror Fusion Program, Lawrence Livermore Laboratory, Rept. UCRL-79532 (1977); prepared for submission to Fiz. Plasmy.

5. W. C. Condit, G. A. Carlson, R. S. Devoto, J. N. Doggett, W. S. Neef, and J. D. Hanson, Preliminary Design Calculations for a Field-Reversed Mirror Reactor, Lawrence Livermore Laboratory, Rept. UCRL-52170 (1976).

6. S. Devoto and J. Hanson, Computation of Properties of a Field-Reversed Mirror Reactor, Lawrence Livermore Laboratory, Rept. UCRL-80220 (1977); prepared for submission to Nucl. Fusion.

7. S. C. Luckhardt and H. H. Fleischmann, "Anomalous Fast-Particle Losses fonm Strong Electron Rings in Quadrupole-Stabilized Mirror Fields," Phys. Rev. Lett. 39, 747 (1977).

8. S. Devoto, Lawrence Livermore Laboratory, private communication (1977).

9. J. A. Byers, Computer Simulation of Field Reversal in Mirror Machines, Phys. Rev. Lett. 39, 1476 (1977).

10. R. L. Post, "On Field Reversal via Neutral Injection," in Proc. Anmual Controlled Fusion Theory Conference, San Diego, CA, 1977 (USERDA, 1977), Paper PB3.

11. B. W. Stallard and M. F. Rensink, Neutral Beam Current Requirements for $\boldsymbol{M} X$, Lawrence Livern ore Laboratory, Rept. UCRL-52205 (1977).

12. D. E. Baldwin, M. F. Rensink, B. I. Cohen, and T. K. Fowler, Lawrence Livermore Laboratory, private communication (1977).

13. D. S. Prono, J. W. Shearer, and R. J. Briggs, "Pulsed lon Diode Experiment," Phys. Rev. Lett. 37, 21 (1976).

14. R. W. Bauer and R. C. Weingart, A Time-Resolved Fast-Neutron Pin Hole Camera for Studying Thermonuclear Plasma, Lawrence Livermore Laboratory, Rept. UCRL-77527 (1976).

1;i. B. G. Logan, J. F. Clauser, F. H. Coensgen, D. L. Correll, W. F. Cummins, C. Gormezano, A. W. Molvik, W. E. Nexsen, T. C. Simonen, B. W. Stallard, and W. C. Turner, "High- $\beta$, Gas-Stabilized, Mirror-Confined Plasma," Phys. Rev. Lett. 37, 1468 (1976). 


\section{APPENDIX. A SIMPLE APPROXIMATION FOR BEAM-TRAPPING EFFICIENCY}

The absorption of energy from the neutral beam by the plasma depends mainly on two interactions. The ionization reaction (cross-section $\sigma_{j}$ ) is

$$
D^{0} \rightarrow D^{+} \rightarrow 2 D^{+}+e^{-}
$$

The charge-exchange reaction (cross-siction $\sigma_{x}$ ) is

$$
D^{0}+D^{+} \rightarrow D^{+}+D^{0}
$$

The estimate of beam-trapping efficiency is complicated by the second reaction because the secondary neutral particle that is produced may interact again by meens of reactions (A1) or (A2). The tertiary neutral may also interact in the same way, and so forth.

As mentioned in the main text, the steady-state code calculations assume that the plasma is "thick," that is, that all of the secondaries, tertiaries, etc. are absorbed. Thus, the total interaction cross-section $\sigma_{\mathrm{T}}$ is used to calculate the absorption parameter $\mathrm{M}$ [Eq. (7)]:

$$
\sigma_{\mathrm{T}}=\sigma_{\mathrm{i}}+\sigma_{\mathrm{x}} \text {. }
$$

In this case, the only escaping neutral particles are those that do not interact. Such an approximation is too optimistic, and needs to be corrected.

On the other hand, the previous study of MFTF neutral-beam injection " used the forward scattering approximation

$$
M \approx \sigma_{i} \int_{r_{\min }}^{r_{\max }} n d \ell .
$$

This equation assumes that reaction (A2) has a negligible effect on the beam; that each secondary neutral is emitted in approximately the same direction as the primary neutral and can be considered a substitute for it. This approximation works best at high energies, but is questionable for the $: \hat{0}-\mathrm{keV}$ beams. We believe that it is too pessimistic, underestimating the amount of absorption of secondary neutrals.

We have formulated a third approximate model for the beam-trapping efficiency. Let $F_{2 M}$ be the interaction fraction of the tangentially injected primary neutral beam:

$$
F_{2 M}=1-e^{-2 M} \text {, }
$$

where $M$ is defined by Eq. ( 7 and the factor 2 is needed because the beam goes in and out of the plasma. Let $f_{i}$ be the fraction that is ionized:

$$
f_{i} \equiv \frac{o_{\mathbf{i}}}{\sigma_{T}} F_{2 M} \text {. }
$$


Then the fraction $f_{x}$ that is charge-exchanged to form a population of secondary neutrals is given by

$$
f_{x} \equiv \frac{\sigma_{T}}{\sigma_{T}} F_{2 M}
$$

The next step is to determine the average path length of the secondary neutrals. An exact calculation would require either elaborate Monte Carlo methods or complicated integrations. We avoid this labor by assuming that the length of the average secondary escape path is only half as long as the length of the primary path because it is born inside the plama and merely has to get out. Thus, for the secondary we take the interaction fraction to be

$$
F_{M}=1-e^{-M}
$$

The ionized and charge-exchanged fractions $\mathrm{f}_{\mathrm{j}}, \mathrm{f}_{\mathrm{x}}^{\prime}$ are then obtained as before:

$$
f_{i}=\frac{\sigma_{i}}{\sigma_{T}} F_{M},
$$

and

$$
\mathbf{f}_{\mathrm{X}}^{\prime}=\frac{\sigma_{\mathrm{x}}}{\sigma_{T}} \mathbf{F}_{\mathbf{M}}
$$

We also ignore the shift in energy between the primary and the secondary and use the same cross sections. Tertiary and higher-order neutrals are approximated in the same manner as the secondaries.

Finally, we sum the ionization fractions in order to find the overall beam-trapping efficiency $\eta$ :

$$
\begin{aligned}
\eta & =f_{i}+f_{x} f_{i}^{\prime}+f_{x}^{\prime} f_{i}^{\prime}+f_{x}^{\prime 2} f_{i}^{\prime}+\ldots \\
& =f_{i}+f_{x} f_{i}^{\prime} \frac{1}{1-f_{x}^{\prime}} .
\end{aligned}
$$

By substituting Egs. (A5) through (A10) into Eq. (11), we then obtain

$$
\eta=\frac{\sigma_{i} F_{2 M}}{\sigma_{T}-\sigma_{X} F_{M}}=\frac{\sigma_{i}\left(1-e^{-2 M}\right)}{\sigma_{i}+\sigma_{x} e^{-M}}
$$

When Eq. (A12) is used, the computed trapping efficiencies are lower than the optimistic predictions of Eq. (A3) and higher than the pessimistic estimates of Eq. (A4); we believe that they are realistic estimates for a pure-hydrogen plasma without impurities. Equation (A12) is therefore the expression for beam-trapping efficiency that we use in the main text. 